Journal of Patient-Centered

$7-27-2020$

\title{
Defining and Characterizing Frequent Attenders: Systematic Literature Review and Recommendations
}

Dip Shukla

Erik Faber

Brian Sick

Follow this and additional works at: https://aah.org/jpcrr

Part of the Community Health and Preventive Medicine Commons, Emergency Medicine Commons, Health Services Research Commons, Medical Humanities Commons, Mental and Social Health Commons, Nursing Commons, Patient Safety Commons, and the Primary Care Commons

\section{Recommended Citation}

Shukla DM, Faber EB, Sick B. Defining and characterizing frequent attenders: systematic literature review and recommendations. J Patient Cent Res Rev. 2020;7:255-64. doi: 10.17294/2330-0698.1747

Published quarterly by Midwest-based health system Advocate Aurora Health and indexed in PubMed Central, the Journal of Patient-Centered Research and Reviews (JPCRR) is an open access, peer-reviewed medical journal focused on disseminating scholarly works devoted to improving patient-centered care practices, health outcomes, and the patient experience. 


\title{
Defining and Characterizing Frequent Attenders: Systematic Literature Review and Recommendations
}

\author{
*Dip M. Shukla, BS, *Erik B. Faber, MS, Brian Sick, MD \\ University of Minnesota Medical School, Minneapolis, MN
}

Purpose To decrease cost and improve efficiency, health care organizations have focused on frequent attenders — patients with high health care utilization. Prior studies have investigated singular health care settings, used varying definitions of frequent attendance, and inconsistently identified factors correlated with frequent attendance. The purpose of this article is to suggest a uniform definition of frequent attenders for different health care settings and to determine factors correlated with frequent attendance.

Methods This systematic review of three databases identified 2761 unique articles; 174 met inclusion criteria. Studies were analyzed for their definition of frequent attenders and factors associated with frequent attendance.

Results Most studies defined frequent attenders by number of health care visits within a set time period $(n=115)$ and top percentile cutoff $(n=42)$. Based on averages across studies, we propose the following frequent attender definitions: for primary care, either the top 10th percentile or at least 10 visits in 12 months; for emergency room, at least 5 visits in 12 months; and for inpatient hospitalization, at least 4 admissions in 12 months. Common factors correlated with frequent attendance were mental health and chronic disease.

Conclusions We propose definitions of frequent attenders for three common health care settings: primary care, emergency room, and inpatient. Future studies should include mental health and chronic disease, among other factors, when studying this population. Adoption of these recommendations will allow comparisons across studies such that meta-analyses may better determine interventions for more appropriate health care utilization. (J Patient Cent Res Rev. 2020;7:255-264.)

Keywords frequent attender; health care utilization; emergency room; primary care; inpatient

$\mathrm{H}$ ealth care organizations are looking for ways to decrease costs and improve the outcomes of their health care settings. ${ }^{1-3}$ Because frequent attenders (FAs), described as those who visit health care settings at a higher rate compared to a general population, use disproportionately more care, ${ }^{4-6}$ this population has been subjected to heightened scrutiny as a target population for potential intervention.

*These authors contributed equally to this work.

Correspondence: Brian Sick, MD,

Department of Internal Medicine, University of Minnesota

Medical School, 420 Delaware Street SE, MMC 741,

Minneapolis, MN 55455 (drbsick@umn.edu)
Multiple systematic studies have been conducted to define this population and determine interventions that best decrease the frequency of their utilization of health care resources. In reviewing this literature, we found a lack of consistency in terminology, study parameters, settings, and targeted outcomes.

Existing systematic reviews tend to focus on one setting or on a specific population ${ }^{4,6-13}$ except for one study, ${ }^{14}$ which looked at frequent users of the health care system as a whole. Many prior reviews comment that FAs are a heterogeneous group ${ }^{4,9,10,13}$ and that there is a need to adopt standard definitions to allow comparisons and generalizability. ${ }^{6,9,10}$ A literature review by Pines et al also suggested the need to identify factors to predict who will become FAs. ${ }^{10}$ 
In an effort to answer these questions, this present systematic review was conducted with the two primary goals of establishing a uniform definition of FAs and determining the factors correlated with frequent attendance. A secondary goal was to determine if the definition varies across different health care settings. By reviewing how FAs were studied in the past, this study can guide the design of future studies in this population.

\section{METHODS}

This systematic review specifically focuses on adult FAs in three health care settings: primary care (PC), emergency room (ER), and inpatient hospitalization (IP). It was conducted in accordance with PRISMA literature review guidelines. ${ }^{15}$

\section{Inclusion and Exclusion Criteria}

Inclusion criteria were primary literature from a refereed journal, containing a "general adult" study population and a definition of frequent attendance, and written in English. Articles were excluded if they were pediatric-only, geriatric-only, or disease-specific population-based studies. Articles focusing on dental care, readmission, dietetic services, and ambulance services/use were excluded to limit problem-oriented studies and guard against nongeneral or otherwise biased adult populations. Abstract-only studies, reviews, editorial/opinion pieces, and posters also were excluded to ensure inclusion of strictly peer-reviewed primary study literature.

\section{Search Strategy and Study Selection}

Ovid MEDLINE ${ }^{\circledR}$ and Epub Ahead of Print, In-Process $\&$ Other Non-Indexed Citations, Daily and Versions ${ }^{\circledR}$ (1946 to August 13, 2019); Embase (1974 to 2019 Week 32); and Cochrane Library (journal beginning to 2016) were searched. No other outside sources, author consultations, or unpublished works were included. All studies were initially title- and abstract-screened (by authors D.S. and E.F.) for relevance and duplicates using the systematic review software program Covidence (Melbourne, Australia). ${ }^{16}$ The full-text, review-eligible articles were coded using Google forms (by authors D.S. and E.F). Articles meeting eligibility were reevaluated jointly (by authors D.S. and E.F.), and studies meeting exclusion criteria were removed. Inclusion disagreements were jointly resolved by both reviewers.
A uniform search strategy was utilized across all three databases. MeSH queries of "primary health care," "exp Emergency Service, Hospital," "Ambulatory care," "ambulatory care facilities/ or outpatient clinics, hospital," "general practice/ or family practice," and "hospital" were used, along with title/abstract/keyword variations of "frequent attend*," "frequent us*," "hot spot*," "high utiliz*," and "super utiliz*" (the asterisk allows incorporation of variable endings to the base word). Search outputs inclusive of both the MeSH and specific title/ abstract/keyword queries were evaluated (onlineonly Supplemental Table S1). The original study end date was March 2016, but the search was rerun in August 2019 with the same end date and with additional terms of "general practice" and "family practice" for a more inclusive search strategy.

\section{Data Extraction}

The coding form extracted publication year, definition of FAs, medical and community settings, nationality of study subjects, study aim, study design, data sources, number and description of FAs and controls in the study, and study metrics associated or not associated with frequent attendance, including whether these factors met statistical significance.

Individual study metrics in each paper were classified into 28 distinct factors, inclusive of an "other" category. During analysis, factors were counted once per study, regardless of the number of individual metrics encompassed within each. Metrics were noted to be diverse in verbiage. For example, metrics of "basic financial needs unmet," "gross income," and "average household income" all described the "income" factor.

\section{Risk of Bias in Individual Studies and Across Studies}

Inclusion/exclusion criteria were strictly applied to investigate the general, adult FA study populations. Results of individual studies were not weighted based on FA sample size to avoid skewing analysis outcomes. Studies were not evaluated for bias with more complex measures due to heterogeneity in study methodology and evolution of outcomes reporting over time. Meeting inclusion/exclusion criteria and publication within a refereed journal was deemed satisfactory for study bias/quality control. 


\section{Summary Measures and Synthesis of Results}

Outcomes of this study are presented as ratios and frequencies. To interrogate if the frequency of factors associated with frequent attendance varied across settings, Pearson's chi-squared test was used to determine statistical significance. To determine a common definition of frequent attendance, onevariable statistical analysis was used to determine the quartile values. Statistical outliers were defined as being 1.5 times the interquartile range (IQR) above the third quartile or below the first quartile.

\section{RESULTS}

\section{Study Characteristics}

The literature search yielded 4358 articles. After 1597 duplicates and 2197 irrelevant articles were excluded, 564 full-text articles were assessed for eligibility. After applying exclusion criteria, 174 articles were included in the analysis. See Figure 1 for PRISMA flowchart results. Pertinent details of and citations for the 174 analyzed studies are shown in online-only Supplemental Table S2.

\section{Publication Rate and Countries of Origin}

Although the first mention of FAs or synonymous terminology was found in peer-reviewed literature published in 1974, the frequency of published FA studies first peaked in the early-to-mid 2000s, with another increase in publications in the early 2010s. The first peak was driven largely by studies focused on non-U.S. populations and the latter driven by U.S. population-focused publications. The non-U.S. publication rate was relatively steady over time, as was the rate of publications that focused on FAs in the PC setting. The medical context may have been a confounding factor in the $2010 \mathrm{~s}$, as the distribution of publication frequency concerning FAs in the ER setting peaked along with that of the U.S. distribution.

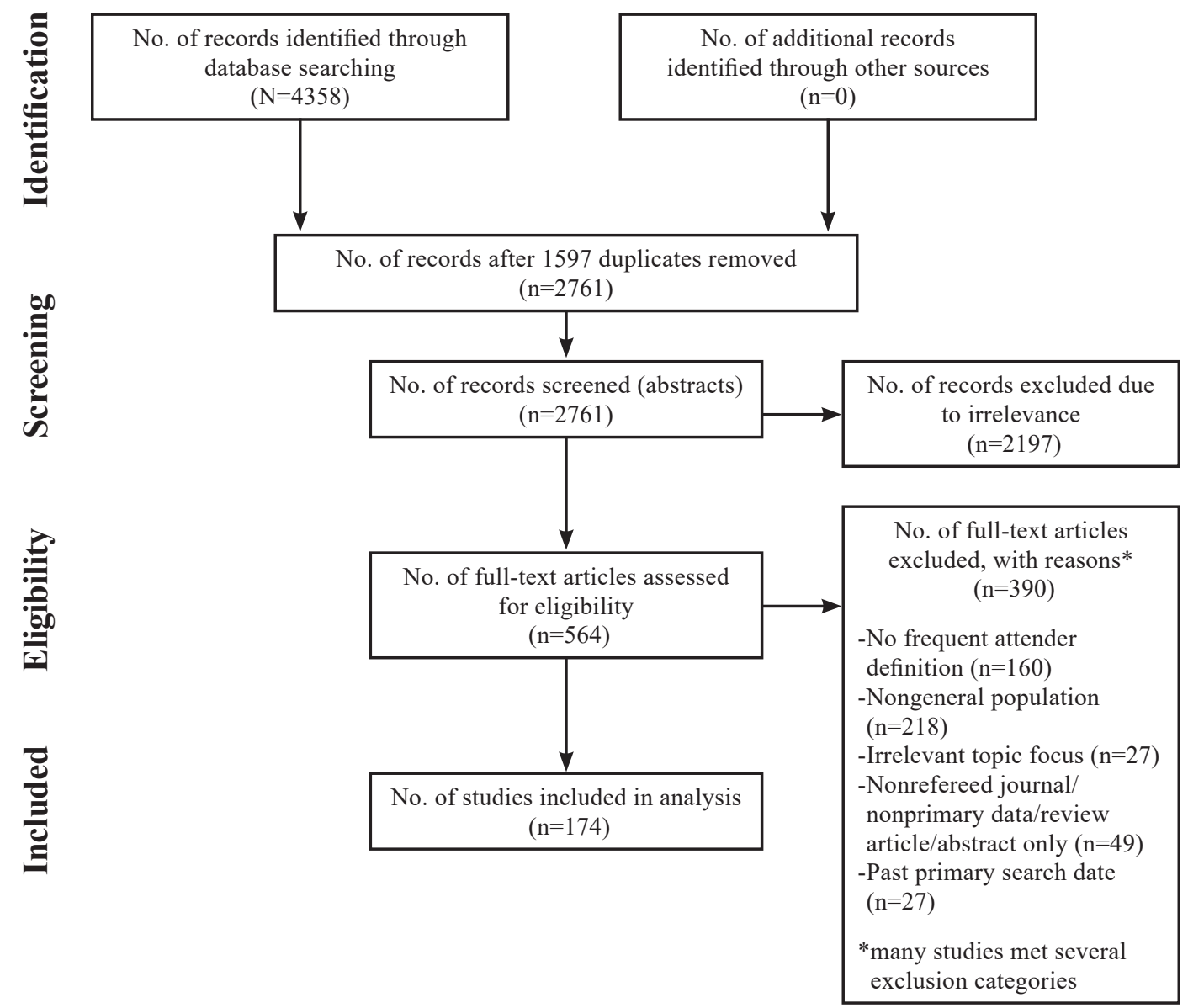

Figure 1. PRISMA flowchart of study selection. 


\section{Study Designs}

Retrospective record reviews and cross-sectional studies were most numerous, comprising $53 \%$ of all study designs. Cohort studies of FAs were found in $34 \%$ of the studies. Case-control, randomized control trial, qualitative assessment, and other study designs were substantially less common. This distribution of study designs was relatively consistent across all medical settings.

\section{Settings}

Of the 174 examined publications, 53\% ( $\mathrm{n}=93)$ studied FAs in the PC context, 45\% $(n=79)$ in the ER context, and $6 \%(n=10)$ in the inpatient context (Figure 2). If a study of FAs was set across multiple medical settings, it was counted in each of those settings. Examining FAs in multiple medical contexts was not uncommon, as this comprised $10 \%$ of the total studies, including $4 \%$ of studies that included medical contexts other than the three categories of PC, ER, or IP. The plurality of FA studies were conducted within the United States $(33 \%, \mathrm{n}=57)$. The remainder were conducted in the United Kingdom, Canada, New Zealand, Australia, and a variety of countries in Europe and Asia. One study occurred in two different countries. ${ }^{17}$ A majority (55\%) of ER studies were from the United States, while only $21 \%$ of all PC studies were from the United States.

A health system-type setting was explicitly described in 101 of the 174 studies (ie, urban, suburban, rural, academic, and/or public community system); of those, 49 described more than one setting. There were more rural-based papers focused on PC, while urban- and academic-based settings seemed to predominate the ER literature.

\section{Number of Frequent Attenders Studied}

Each study varied greatly in terms of study population size and number of FAs it included. The median number of FAs studied was between 105 and 262, depending on the medical context. The third quartile for each context

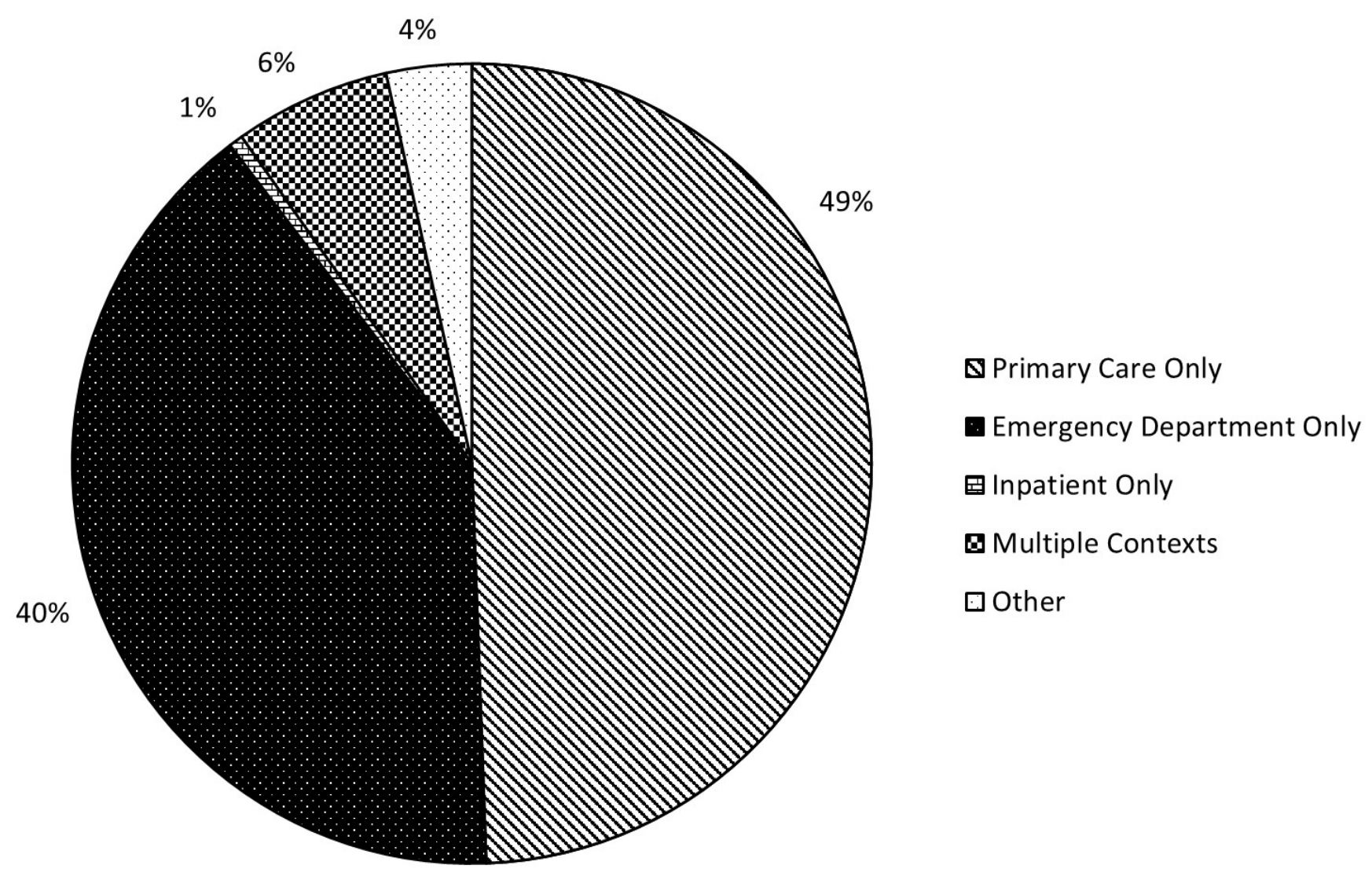

Figure 2. Distribution of medical contexts of the 174 examined studies. "Other" contexts were those that included atypical descriptors like "specialty clinics" in addition to at least one traditional context (primary care, emergency department, or inpatient). 
was about 10-fold higher than the median, and the first quartile was less than half the median. Additionally, there were 25 studies in which the number of FAs was at least 2.5 times above the IQR. These studies were often conducted in populations from national health care databases.

\section{Definition of a Frequent Attender}

The most common way studies defined FAs was based on how often a patient appeared to a certain medical setting. A "number of visits per year" definition was used in $115(66 \%)$ studies (9 additional studies combined this with other definitions), whereas a "top X percentile" definition was used in 42 (24\%) studies (Figure 3). Cost and a combination of definitions were less commonly used for the remaining 8 articles. Seven studies further defined FAs by "top $\mathrm{X}$ utilizer of health care in a population" (eg, top 500 utilizers in a population of 10,000$)$. More than $40 \%$ of all definitions were multifactorial in that they controlled for or considered aspects such as age and gender as a component of the definition. Finally, several studies commented on how they would define a FA but did not study a discrete population.

The definition of a FA varied based on the medical context examined. Within the PC setting, an equal number of studies used number of visits per year (47\%) and top X percentile (47\%) definition. Within the ER context, $88 \%$ of studies used number of visits per year. Of the 12 IP studies examined, 6 used number of visits per year, 3 defined a FA by the costliest patients, and 3 used top X percentile.

For each medical context, the quantitative distribution of FA definitions was examined across studies. For studies that defined FAs by the number of visits in a certain time, this number was standardized to number of visits per year (Figure 4). For the PC studies using number of visits $(n=47)$, the median was 10 visits per year, and the 25th-75th percentile (IQR) of definitions ranged from 8 to 12 visits per year. Five studies had a definition that was a statistical outlier above the maximum: 20, 24, 24, 27, and 144 visits per year. Additionally, 2 PC studies were excluded from this analysis because one used the number of physicians seen instead of number of visits and another used the number of ER visits instead of the number of PC visits. For the ER studies using number of visits $(n=71)$, the median definition of FAs was 5 visits per year (IQR: 4-6). Thirteen studies had a definition that was a statistical outlier above the maximum: 10, 10, $10,10,10,12,12,12,12,15,20,20$, and 36 visits per year. Two ER studies were excluded from this analysis because one did not specify a quantifiable definition and another used an array of definitions but

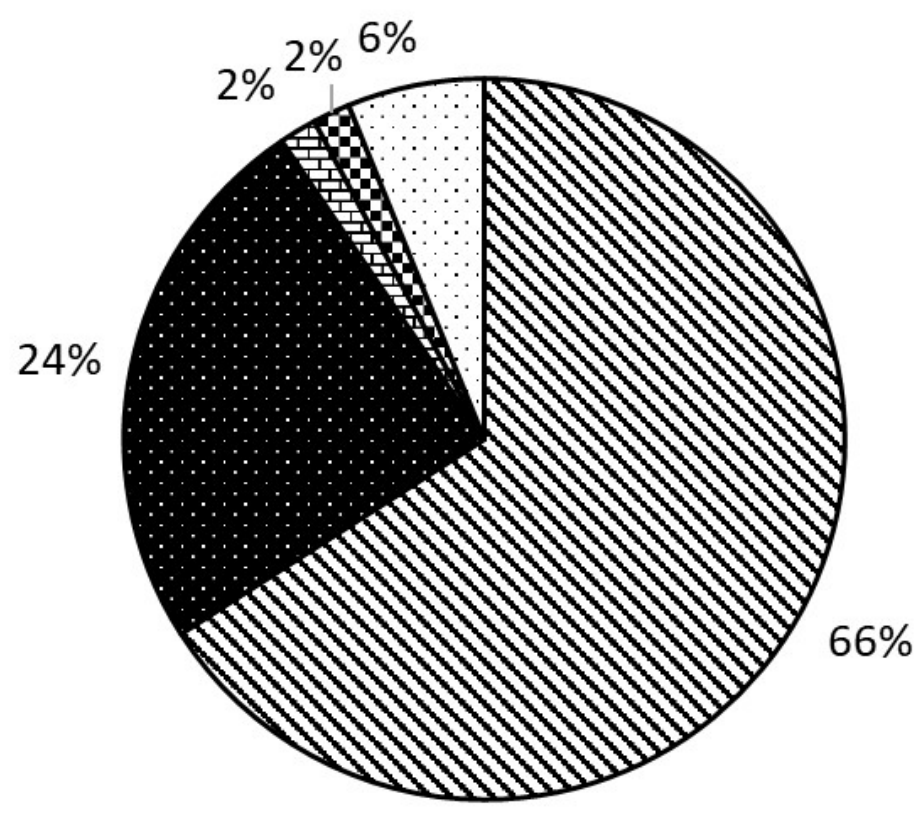

\author{
$\boldsymbol{\Delta N u m b e r}$ of Visits / Year \\ Dercentile \\ 由 Cost \\ OOther \\ $\square$ Combination of Categories
}

Figure 3. Frequent attender definition categories. 


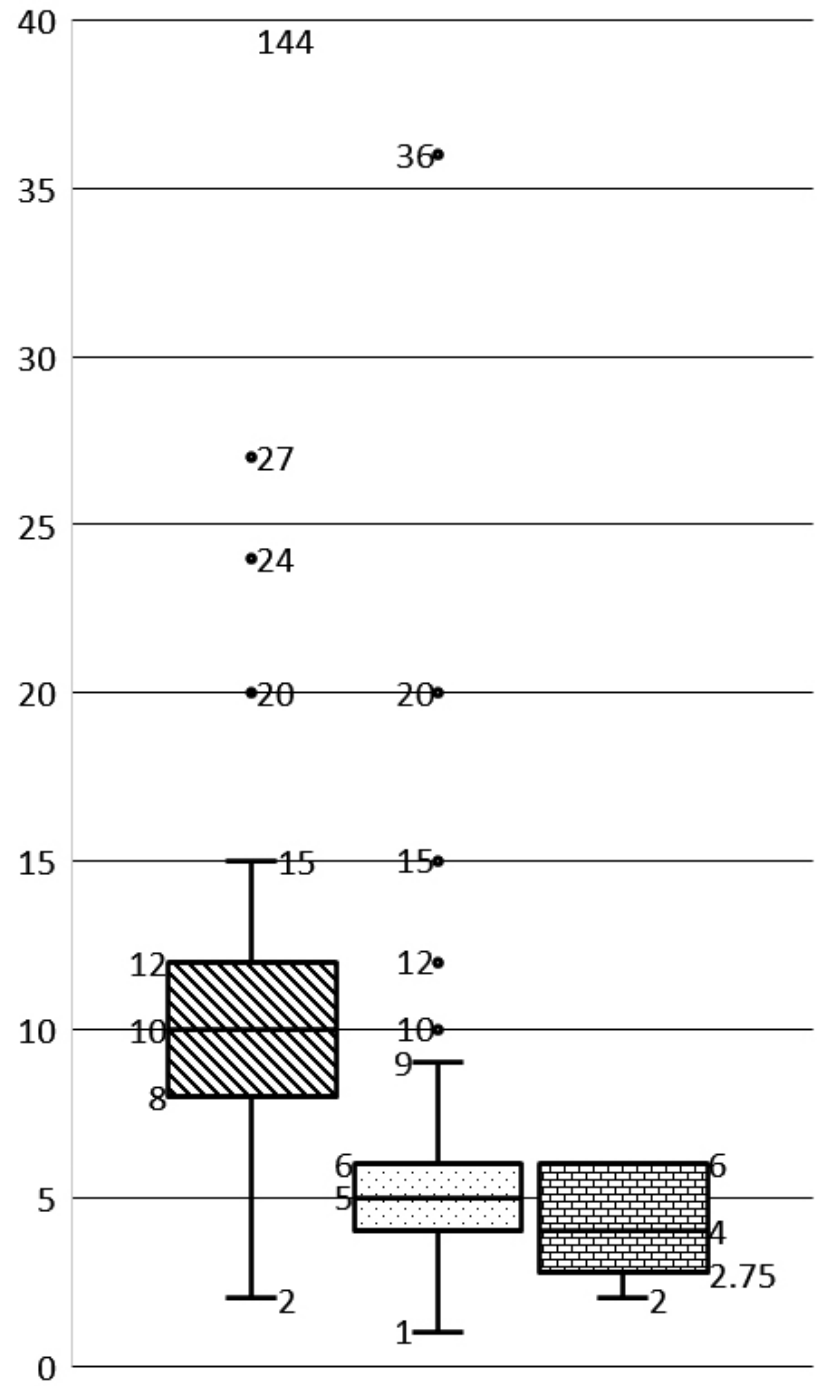

did not show any preference. For the studies that used a tiered definition of frequent attendance, the lowest FA definition was used (eg, the FA definition was used over the "very FA" or "super FA" definition). For the IP studies, 6 used number of visits per year to define FAs. Those definitions were 2, 3, 4, 4, 6, and 6 visits to an IP setting in 12 months to qualify as FAs. The IP median FA definition was 4 visits per year (IQR: 2.75-6).

Studies that used top percentile of health care utilizers to define FA were also compared. For PC studies $(n=49)$, the median, upper, and lower quartiles were the top $10 \%$ of utilizers. Therefore, all other definitions were statistical outliers, with 20 values ranging from the top $2.5 \%$ of utilizers to the top $40 \%$. The ER studies did not often use top percentile to define FAs $(n=6)$,

\section{$\Delta$ Primary Care \\ DEmergency Room}

口Inpatient
Figure 4. Distribution of frequent attender definitions by number of visits per year as well as by primary care $(n=47)$, emergency room ( $n=73)$, and inpatient study settings $(n=6)$ that used number of visits as a metric of frequent attendance. All definitions were normalized to number of visits per year. The top of each box is the third quartile value, the middle line the median, and the bottom line the first quartile value. The whiskers represent the top and bottom of the data range, excluding statistical outliers, which are represented as points on the graph. but of those that did, the median definition was the top $7.5 \%$ of utilizers (IQR: top $3.025 \%-21.25 \%$ ). In the IP studies, 2 studies used top percentile of utilizers to define FAs: top $2.5 \%$ and top $10 \%$.

\section{Factors Used to Examine or Predict Frequent Attendance}

We also collected the factors each study used to examine frequent attendance and how often the factor was correlated with frequent attendance (Table 1). The number of studies that examined a factor and found it to be statistically correlated (with or without statistical certainty) and not correlated are shown in onlineonly Supplemental Table S3. IP studies were not included due to their low prevalence relative to studies examining FAs in the ER and PC contexts. 
Table 1. Prevalence of Examined Factors Shown to Correlate With Frequent Attendance

\begin{tabular}{|c|c|c|}
\hline $\begin{array}{l}\text { Factor (no. of studies } \\
\text { mentioning) }\end{array}$ & $\begin{array}{c}\text { No. of } \\
\text { studies } \\
\text { examining } \\
\text { correlation }\end{array}$ & $\begin{array}{c}\text { Positively } \\
\text { correlated } \\
\text { studies* }\end{array}$ \\
\hline Gender (152) & 83 & $57 \%$ \\
\hline Age (149) & 88 & $67 \%$ \\
\hline Mental health (103) & 83 & $89 \%$ \\
\hline $\begin{array}{l}\text { Marital status/Family } \\
\text { metrics }(67)\end{array}$ & 54 & $50 \%$ \\
\hline Drug/Substance abuse (65) & 48 & $77 \%$ \\
\hline Chronic disease (53) & 42 & $88 \%$ \\
\hline Employment (53) & 39 & $79 \%$ \\
\hline Education (45) & 33 & $70 \%$ \\
\hline Race/Ethnicity (43) & 20 & $70 \%$ \\
\hline Insurance status/type (38) & 23 & $83 \%$ \\
\hline Housing status (37) & 25 & $48 \%$ \\
\hline Social situation/support (35) & 27 & $74 \%$ \\
\hline Diagnoses (33) & 18 & $83 \%$ \\
\hline $\begin{array}{l}\text { Multiple diseases/ } \\
\text { comorbidities (33) }\end{array}$ & 24 & $83 \%$ \\
\hline Income (27) & 20 & $80 \%$ \\
\hline $\begin{array}{l}\text { Primary care provider } \\
\text { status (19) }\end{array}$ & 9 & $56 \%$ \\
\hline $\begin{array}{l}\text { Prescription-based } \\
\text { metrics (18) }\end{array}$ & 14 & $86 \%$ \\
\hline Acuity/Triage (16) & 10 & $70 \%$ \\
\hline Perceived health (15) & 11 & $100 \%$ \\
\hline Socioeconomic status (15) & 8 & $50 \%$ \\
\hline Persistent overutilization (14) & 10 & $60 \%$ \\
\hline Distance-based metrics (13) & 13 & $69 \%$ \\
\hline $\begin{array}{l}\text { Use of other medical } \\
\text { settings (12) }\end{array}$ & 8 & $100 \%$ \\
\hline Severity (11) & 9 & $89 \%$ \\
\hline Chief complaint (9) & 1 & $100 \%$ \\
\hline Medical disposition (6) & 3 & $100 \%$ \\
\hline Disability (4) & 2 & $100 \%$ \\
\hline Other (120) & 114 & $72 \%$ \\
\hline
\end{tabular}

*Percentage among studies that reported any correlation.

Of the top 10 most commonly examined factors (range of 38-152 studies that mention the factor), mental health was most correlated with frequent attendance ( $89 \%$ of studies reported positive correlation), followed by chronic disease (88\%), insurance status/type (83\%), employment (79\%), and drug/substance abuse $(77 \%)$ (Table 1). Furthermore, chi-squared tests were performed to compare if these factors were differently correlated across medical settings or different countries. No factors were found to have a significant correlative difference across settings or countries with a cutoff value of $\mathrm{P}<0.05$ (data not shown). It is important to note that 120 of the 174 publications also included factors that were grouped in an "Other" category.

\section{DISCUSSION}

This review of 174 publications was conducted to fill gaps identified by other systematic reviews. Specifically, we propose a unified definition of FAs for multiple health care settings based on the literature. To further help guide future studies, we also determined the risk factors correlated with frequent attendance.

Based on our review, we propose the adoption of universal definitions of FAs by medical context. Context-specific definitions will improve cross-study comparisons and understanding of FA populations. For the PC context, either the top $10 \%$ of health care users or at least 10 visits to an outpatient setting in 12 months is appropriate. By identifying the top 10th percentile of health care users, a more population-based approach may be taken. However, the use of a percentile as the standard definition requires retrospective review of utilization and may limit the ability to intervene in a timely manner on behalf of FAs. For the ER context, we propose defining FAs as visiting at least 5 times in 12 months. This definition is similar to one proposed by Locker et al of at least 4 times in 12 months, which was based on a statistical calculation that $99.99 \%$ of people would be expected to present less than 4 times per year. ${ }^{18}$ Lastly for the IP context, we propose a FA definition of at least 4 admissions in 12 months.

In the literature, FA definitions were often based on prior studies and, generally, sought to identify the highestutilizing patients. We based our proposed definitions on those we found to be the most utilized, with the hope of unifying the approach of future works. While many studies instituted additional qualifications for gender and age groups to identify specific subpopulations of FAs, we propose adopting the minimums above and adding additional definition parameters as appropriate for the study being conducted. Doing so may stratify 
populations based on varying reasons for attendance and not necessarily target consecutive highest-utilizing individuals.

In evaluating the factors correlated with frequent attendance, we noted many similarities in the use of specific factors across publications regardless of medical context or nation of origin. Gender, age, mental health, marital status/family metrics, drug/ substance abuse, chronic disease, employment, and education level were commonly studied. The two most well-examined factors that were best correlated with frequent attendance were mental health $(89 \%)$ and chronic disease (88\%). This is not surprising since arguably our health care system struggles most to adequately treat these two disease classes, in addition to the overall high prevalence of mental health and chronic disease within the general population. ${ }^{19,20}$ Interestingly, some commonly examined factors, like gender, marital status, and housing status, were not often correlated with frequent attendance. This latter factor is especially surprising, as homelessness is historically thought to be a risk factor for overuse of health care contexts like the ER. ${ }^{21-23}$

Upon examining every reported frequent attendanceassociated risk factor, we found no significant difference $(\mathrm{P}<0.05)$ between the PC, ER, and combination of both contexts, as well as no significant difference between studies from the United States and studies abroad. This was true despite the observation that U.S. studies disproportionately focused on the ER setting while studies abroad focused more on PC.

\section{Limitations}

While we believe our analysis is comprehensive and ultimately useful, there are limitations to our work. This study is limited to the general adult population. Pediatric and geriatric FAs might have different risk factors that predict their health care use. Studies that focused on disease-specific populations (eg, sickle cell or mental health) were excluded. Publication bias might skew the results toward studies that found positive or significant correlations. Other biases in this analysis include the exclusion of non-English papers and a loose definition adopted for statistical significance. Any statistics calculated on the data was accepted as valid independent of methodology used.
For a study to claim a "correlation" with a certain risk factor, the study simply had to report this association. No numerical evidence was necessary, and only about half the studies included a statistical analysis. Future studies should strive to include more rigorous statistical analysis. Finally, we believe our results to be generalizable; however, we recognize that every setting is unique, and it will be incumbent on those helping FAs at a certain location to critically determine how these findings apply to their patient population.

It should also be noted that our understanding of frequent attendance is limited by past publications. Other important contributing factors, such as health care literacy and access, may not have been captured within our study. ${ }^{24}$ Due to their complex, heterogeneous nature, these factors are difficult to measure but critical to the generation of patient care measures. Future avenues of work may include studying combinations of identified factors using retrospective database analysis to elucidate their strength of correlation with frequent attendance or using prospective analysis of patients with multiple identified factors to see if their attendance is increased compared to patients with only one factor.

\section{Research Implications}

Despite these limitations, this investigation unifies disparate studies into a generalized understanding of frequent attendance. It also identifies studied factors that may help institutions better understand this atrisk population to effect appropriate interventions. Consistent use of a uniform definition for FAs will facilitate comparisons across studies such that pooled data and meta-analyses could help determine the best interventions for guiding more appropriate utilization of health care resources. Additionally, consistent inclusion of the factors associated with high utilization, such as mental health, chronic disease, insurance status, and drug/substance use, can ensure that these factors are accounted for in the analysis of the data about effective interventions. From a managed care lens, adopting a uniform definition for FAs could also help determine the utility and applicability of such a metric across settings and over time better serve patients and use health care resources more efficiently. While the definition of an FA necessitates some passage of time to allow observation of high 
utilization, studying FAs retrospectively can be helpful in managing future care of FAs. The pace at which utilization data is becoming accessible may change the utility of retrospective definitions and facilitate more rapid interventions.

An accurate determination of individual- and population-level risk for health services use has great potential for modifying reimbursement structures of managed care organizations with more inclusive and rigorous modeling. Currently, common methods center on diagnosis-related groups to define patient complexity. The case mix index and hierarchical condition category (HCC) risk adjustment provide a basis for comparing disease severity and clinical complexity of treated populations. The HCC also allows prospective estimation of future resource intensiveness. However, these are limited by model inclusion metrics. As value-based reimbursement structures continue to grow, incorporating additional elements associated with and predictive of frequent attendance can improve allocation of financial resources and health equity. ${ }^{25} \mathrm{~A}$ uniform definition and standardized evaluation of FAs is necessary to use these metrics in such value-based reimbursements.

Certainly, downstream efforts will not only require a unified understanding of past efforts, as proposed here, but necessitate new interventions and measures to allow a more holistic, actionable understanding of frequent attendance.

\section{CONCLUSIONS}

Based on examination of the multiple definitions of frequent attenders across settings, the applicability and need for universal definitions per setting is evident. In this systematic review, we sought a common definition for FAs in various practice settings and a characterization of factors associated with frequent attendance. We identified several common factors frequently correlated with frequent attendance, such as mental health and chronic disease, but also demonstrated a lack of reliable, consistent factors across studies. Herein, we propose FA definitions for the primary care, emergency room, and hospital inpatient settings. Adoption of these definitions will improve health care communities' ability to study, appropriately intervene, and care for this vulnerable population.

\section{Patient-Friendly Recap}

- The ability of health systems to support patients with high health care utilization, known as frequent attenders, is hindered by a lack of understanding of this population.

- Different health care settings are unique in their use of resources and, therefore, recognize different levels of patient utilization as excessive.

- The authors reviewed published studies from primary care, emergency room, and hospital inpatient settings to recommend universal cutoffs for defining frequent attendance.

- They also found that mental health and chronic disease were patient factors associated with being a frequent attender.

\section{Acknowledgments}

The authors thank University of Minnesota Medical School library staff Sarah Jane Brown, Amy Claussen, and Dawn Hackman for their support in the development of the search strategy and execution of the database searches.

\section{Author Contributions}

Study design: all authors. Data acquisition or analysis: all authors. Manuscript drafting: all authors. Critical revision: all authors.

\section{Conflicts of Interest}

None.

\section{Funding Sources}

Erik Faber received support to do this work from a National Institutes of Health/National Institute of General Medical Sciences award (\#T32GM008244).

\section{References}

1. Cowan RM, Trzeciak S. Clinical review: emergency department overcrowding and the potential impact on the critically ill. Crit Care. 2005;9:291-5. CrossRef

2. Ruger JP, Richter CJ, Spitznagel EL, Lewis LM. Analysis of costs, length of stay, and utilization of emergency department services by frequent users: implications for health policy. Acad Emerg Med. 2004;11:1311-7. CrossRef

3. Handel DA, McConnell KJ, Wallace N, Gallia C. How much does emergency department use affect the cost of Medicaid programs? Ann Emerg Med. 2008;51:614-21. CrossRef

4. LaCalle E, Rabin E. Frequent users of emergency departments: the myths, the data, and the policy implications. Ann Emerg Med. 2010;56:42-8. CrossRef

5. Neal RD, Heywood PL, Morley S, Clayden AD, Dowell AC. Frequency of patients' consulting in general practice and workload generated by frequent attenders: comparisons between practices. Br J Gen Pract. 1998;48:895-8. 
6. Vedsted P, Christensen MB. Frequent attenders in general practice care: a literature review with special reference to methodological considerations. Public Health. 2005;119:118-37. CrossRef

7. Althaus F, Paroz S, Hugli O, et al. Effectiveness of interventions targeting frequent users of emergency departments: a systematic review. Ann Emerg Med. 2011;58:41-52. CrossRef

8. Kumar G, Klein R. Effectiveness of case management strategies in reducing emergency department visits in frequent user patient populations: a systematic review. J Emerg Med. 2013;44:717-29. CrossRef

9. Moe J, Kirkland S, Ospina MB, et al. Mortality, admission rates and outpatient use among frequent users of emergency departments: a systematic review. Emerg Med J. 2016;33:230-6. CrossRef

10. Pines JM, Asplin BR, Kaji AH, et al. Frequent users of emergency department services: gaps in knowledge and proposed research agenda. Acad Emerg Med. 2011;18:e64-9. CrossRef

11. Soril LJ, Leggett LE, Lorenzetti DL, Noseworthy TW, Clement FM. Reducing frequent visits to the emergency department: a systematic review of interventions. PLoS One. 2015;10(4):e0123660. CrossRef

12. Smits FT, Wittkampf KA, Schene AH, Bindels PJ, Van Weert HC. Interventions on frequent attenders in primary care: a systematic literature review. Scan J Prim Health Care. 2008;26:111-6. CrossRef

13. van Tiel S, Rood PP, Bertoli-Avella AM, et al. Systematic review of frequent users of emergency departments in non-US hospitals: state of the art. Eur J Emerg Med. 2015;22:306-15. CrossRef

14. Tricco AC, Antony J, Ivers NM, et al. Effectiveness of quality improvement strategies for coordination of care to reduce use of health care services: a systematic review and meta-analysis. CMAJ. 2014;186(15):E568-78. CrossRef

15. Moher D, Liberati A, Tetzlaff J, Altman DG; Prisma Group. Preferred reporting items for systematic reviews and meta-analyses: the PRISMA statement. PLoS Med. 2009;6(7):e1000097. CrossRef
16. Macdonald M, Misener RM, Weeks L, Helwig M. Covidence vs Excel for the title and abstract review stage of a systematic review. Int J Evid Based Healthc. 2016;14:200-1. CrossRef

17. Dowrick CF, Bellón JA, Gómez MJ. GP frequent attendance in Liverpool and Granada: the impact of depressive symptoms. Br J Gen Pract. 2000;50:361-5.

18. Locker TE, Baston S, Mason SM, Nicholl J. Defining frequent use of an urban emergency department. Emerg Med J. 2007;24:398-401. CrossRef

19. Whiteford HA, Degenhardt L, Rehm J, et al. Global burden of disease attributable to mental and substance use disorders: findings from the Global Burden of Disease Study 2010. Lancet. 2013;382:1575-86. CrossRef

20. Bodenheimer T, Chen E, Bennett HD. Confronting the growing burden of chronic disease: Can the U.S. health care workforce do the job? Health Aff (Millwood). 2009;28:64-74. CrossRef

21. Han B, Wells BL. Inappropriate emergency department visits and use of the Health Care for the Homeless Program services by homeless adults in the northeastern United States. J Public Health Manag Pract. 2003;9:530-7. CrossRef

22. Brickner PW, Scanlan BC, Conanan B, et al. Homeless persons and health care. Ann Intern Med. 1986;104:405-9. CrossRef

23. O'Toole TP, Gibbon JL, Hanusa BH, Fine MJ. Preferences for sites of care among urban homeless and housed poor adults. $J$ Gen Intern Med. 1999;14:599-605. CrossRef

24. Berkman ND, Sheridan SL, Donahue KE, Halpern DJ, Crotty K. Low health literacy and health outcomes: an updated systematic review. Ann Intern Med. 2011;155:97-107. CrossRef

25. Ash AS, Mick EO, Ellis RP, Kiefe CI, Allison JJ, Clark MA. Social determinants of health in managed care payment formulas. JAMA Intern Med. 2017;177:1424-30. CrossRef

(C) 2020 Aurora Health Care, Inc. 